\title{
Traction on the Retina Induced by Saccadic Eye Movements in the Presence of Posterior Vitreous Detachment
}

\author{
Colangeli E. ${ }^{*, 1}$, Repetto $R^{1,2}$, Tatone $A .^{1}$ and Testa $A .^{1}$ \\ ${ }^{1}$ DISAT - Department of Engineering of Structures, Water and Soil - University of L'Aquila, L'Aquila - Italy \\ ${ }^{2}$ Department of Bioengineering - Imperial College, London - UK \\ *Corresponding author: DISAT - Facoltà di Ingegneria, Piazzale Pontieri 1, 67040 Monteluco di Roio (AQ) - \\ Italy; elisa.c@ing.univaq.it
}

\begin{abstract}
Vitreous humor, which fills most part of the eye's interior, is a gellike substance in the young healthy man. With aging, it undergoes a liquefaction process that usually leads to vitreous separation from the retina, which is called Posterior Vitreous Detachment (PVD). Sites of strong adherence between the vitreous and the retina may prevent a complete detachment. In such circumstances the adherent vitreous fibers may pull so hard as to produce a retinal break. This rupture mechanism may possibly be induced by eye rotations. Our aim is to study the dynamic stress concentration on the retina induced by saccadic eye movements. While our formulation applies to a general three-dimensional problem, we consider a plane strain problem just to simplify the computational setting. The solid vitreous is modeled as a hyperelastic incompressible solid, endowed with a MooneyRivlin response function, and the liquefied vitreous as a Newtonian fluid. Moreover, we account for finite-amplitude eye rotations. We implemented our model in Comsol by using the PDE Application Mode in weak form and the Moving Mesh Application Mode. The numerical simulations show that very high values of the traction are attained at the boundary of the interface during eye rotations.
\end{abstract}

Keywords: Vitreous body, Posterior Vitreous Detachment, Mooney-Rivlin, FluidStructure Interaction.

\section{Introduction}

The vitreous body is the largest single structure of the eye, making up approximately $80 \%$ of its total volume. It fills the vitreous cavity and is delimited anteriorly by the lens and posteriorly by the retina. The mature vitreous body is an avascular, transparent gel, with viscoelastic rheological properties (1, 2), containing more than $99 \%$ water and including, for the remaining part, solid structural components (collagen, hyaluronic acid and non-collagenous proteins).

Substantial rheologic, biochemical and structural changes occur in the vitreous during aging (4; 5). Typically after the fourth decade of life, a progressive liquefaction of the vitreous structure is observed, associated with changes in the chemical state and conformation of the component macromolecules. Alterations in the normal hyaluronic acid-collagen association result indeed in the simultaneous formation of large liquid vitreous pockets (synchysis), clinically recognized as lacunae, and aggregation of densely packed collagen fibrils into bundles of parallel macroscopic fibers (syneresis). The liquefaction process normally originates in the center of the vitreous chamber, and may extend toward peripheral areas, eventually leading to a global collapse of the gel structure. Shortening and condensation of vitreous collagen fibrils could contract the vitreous body and pull the posterior vitreous. In addition, the physiological weakening of the adhesion (8) between the outermost part of the vitreous, the cortex, and the inner layer of the retina at the posterior pole, allows the liquid vitreous to enter the retro-cortical space, progressively causing the separation of the posterior vitreous cortex from of the retina. Such a process is known as Posterior Vitreous Detachment (PVD). In most cases PVD is not sight-threatening, it leaves sight abilities unchanged and it may progress as much 
as to induce the complete detachment of the vitreous cortex from the retina. However, strong adhesion between the vitreous and the retina may localize at certain sites, inhibiting the complete vitreous detachment. In such circumstances, during rapid rotational eye movements (saccadic movements), the liquid vitreous may act as a wedge in the retro-cortical space further separating the cortex from the retina. The high traction forces originated by such a motion on the boundary of the adhesion areas may generate tears on the retina. Even a small retinal tear may then allow fluid to flow from the vitreous cavity into the sub-retinal space, thus triggering a retinal detachment.

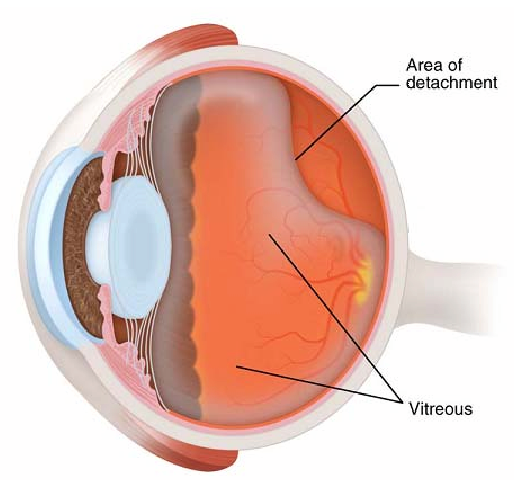

Figure 1: The vitreous chamber with Posterior Vitreous Detachment.

The aim of this work is to describe from a mechanical point of view the dynamics of the detached vitreous induced by eye rotations. By means of numerical simulations we can obtain estimates of the traction that a partially detached vitreous may exert on the retina. The importance of this issue is stressed by Sebag in (4):

How to best evaluate the presence or absence of vitreous traction and how to quantitate the degree of vitreous traction is presently not known.

\section{The Mathematical Model}

We state the problem in a two dimensional setting accounting for a plane strain problem and we consider a configuration of the vitreous after PVD, where the vitreous chamber, which is assumed to be rigid, is partially filled with the detached vitreous, still having a gel-like consistence, and partially with the liquefied vitreous. The liquefied vitreous is modeled as a Newtonian incompressible fluid, and the gel-like vitreous as a homogeneous isotropic, non-linear hyperelastic incompressible solid, characterized by a Mooney-Rivlin strain energy function. We study the response to finite-amplitude (saccadic) eye rotations while assuming that the outermost layer of the eye (sclera) is rigid. We assume as a balance principle for a Cauchy continuum that in any shape $\mathfrak{D}$, the working

$$
\int_{\mathfrak{D}} \mathbf{b} \cdot \mathbf{w} d V+\int_{\partial \mathfrak{D}} \mathbf{t} \cdot \mathbf{w} d A-\int_{\mathfrak{D}} \mathbf{T} \cdot \nabla \mathbf{w} d V
$$

be zero for any test velocity field w. Herein, $\mathbf{b}$ is the volume force distribution in the actual shape $\mathfrak{D}, \mathbf{t}$ is the surface force distribution on the boundary $\partial \mathfrak{D}$ and $\mathbf{T}$ is the Cauchy stress tensor field. The balance principle may be conveniently restated by pulling-back all fields to a fixed paragon shape $\mathcal{D}$, thus transforming (1) into

$$
\int_{\mathcal{D}} \mathrm{b} \cdot \mathrm{w} d V+\int_{\partial \mathcal{D}} \mathrm{t} \cdot \mathrm{w} d A-\int_{\mathcal{D}} \mathrm{s} \cdot \nabla \mathrm{w} d V
$$

where $\mathbf{S}:=\mathbf{T} \mathrm{F}^{-\mathbf{T}}$ det $\mathrm{F}$ is the Piola-Kirchhoff stress tensor.

Before characterizing the solid and the fluid materials, the balance principle can be detailed in order to emphasize the role of the interface tractions. This is convenient even on the computational side, as will be explained later. Let us split $\mathfrak{D}$ into two parts $\mathfrak{D}_{s}$ and $\mathfrak{D}_{f}$, corresponding to the domains occupied respectively by the solid and the fluid, and denote by $\partial_{\star} \mathfrak{D}_{s}$ and $\partial_{\star} \mathfrak{D}_{f}$ their exterior boundaries and by $\mathfrak{I}$ the interface between the two parts. If we look at this composite continuum as a whole, we can restate the balance principle requiring that the working

$$
\begin{aligned}
\int_{\mathfrak{D}} \mathbf{b} \cdot \mathbf{w} d V+\int_{\partial_{\star} \mathfrak{D}} \mathbf{t} \cdot \mathbf{w} d A+ \\
\quad-\int_{\mathfrak{D}} \mathbf{T} \cdot \nabla \mathbf{w} d V-\int_{\mathfrak{I}} \mathbf{t}^{*} \cdot \llbracket \mathbf{w} \rrbracket d A
\end{aligned}
$$

be zero for any test velocity field $\mathbf{w}$ allowed to be discontinuous at $\mathfrak{I}$. The last term in (3) accounts for a discontinuity of the test velocity field at the interface, while $\mathbf{t}^{*}$ is the interface stress. In the following subsections, the balance principles for fluid and solid parts are separately considered. 


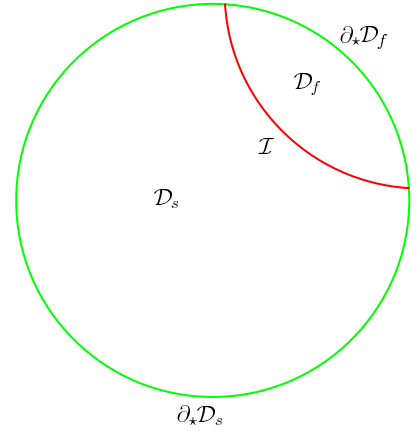

Figure 2: Geometry of the paragon shape $\mathfrak{D}$.

\subsection{The solid}

The balance principle for the fluid part states that the working

$$
\begin{gathered}
\int_{\mathfrak{D}_{s}} \mathbf{b}_{s} \cdot \mathbf{w}_{s} d V+\int_{\partial_{\star} \mathfrak{D}_{s}} \mathbf{t}_{s} \cdot \mathbf{w}_{s} d A+ \\
+\int_{\mathfrak{I}} \mathbf{t}_{s} \cdot \mathbf{w}_{s} d A-\int_{\mathfrak{D}_{s}} \mathbf{T}_{s} \cdot \nabla \mathbf{w}_{s} d V
\end{gathered}
$$

be zero for any test velocity field $\mathbf{w}_{s}$ on the actual shape $\mathfrak{D}_{s}$. It is convenient to rewrite (4) in the form (2) thus requiring that the working

$$
\begin{array}{r}
\int_{\mathcal{D}_{s}} \mathrm{~b}_{s} \cdot \mathrm{w}_{s} d V+\int_{\partial_{\star} \mathcal{D}_{s}} \mathrm{t}_{s} \cdot \mathrm{w}_{s} d A+ \\
+\int_{\mathcal{I}} \mathrm{t}_{s} \cdot \mathrm{w}_{s} d A-\int_{\mathcal{D}_{s}} \mathrm{~S}_{s} \cdot \nabla \mathrm{w}_{s} d V
\end{array}
$$

be zero for any test velocity field $\mathrm{w}_{s}$ on the paragon shape. Let us consider the restriction of the deformation gradient $F$ to plane deformations, whose matrix representation will be

$$
[\mathrm{F}]=\left(\begin{array}{ccc}
f_{11} & f_{12} & 0 \\
f_{21} & f_{22} & 0 \\
0 & 0 & 1
\end{array}\right)
$$

The principal invariants of the right CauchyGreen strain tensor $C:=F^{\top} F$ are

$$
\begin{aligned}
& I_{1}:=\operatorname{tr} \mathrm{C}=\mathrm{F} \cdot \mathrm{F}, \\
& I_{2}:=\frac{1}{2}\left[(\operatorname{tr} \mathrm{C})^{2}-\operatorname{tr}^{2}\right]=\operatorname{cof}(\mathrm{F}) \cdot \operatorname{cof}(\mathrm{F}) .
\end{aligned}
$$

As we consider an incompressible material, the principal invariants are assumed to be evaluated along plane isochoric motions. The general form of the MooneyRivlin strain energy function is

$$
W(\mathrm{~F})=c_{10}\left(I_{1}-3\right)+c_{01}\left(I_{2}-3\right),
$$

in which $c_{10}$ and $c_{01}$ are material constants. For plane deformations $I_{1}=I_{2}$ and the above expression simplifies to

$$
W(\mathrm{~F})=\left(c_{10}+c_{01}\right)\left(I_{1}-3\right) .
$$

Since the strain energy rate can be expressed as

$$
\dot{W}=\mathrm{S}_{s} \cdot \dot{\mathrm{F}},
$$

by differentiating (8) we obtain

$$
\dot{W}=\left(c_{10}+c_{01}\right) \dot{I}_{1}=2\left(c_{10}+c_{01}\right) \mathrm{F} \cdot \dot{\mathrm{F}} .
$$

Hence, the response for the Piola-Kirchhoff stress tensor on the paragon shape $\mathcal{D}_{s}$ turns out to be

$$
\mathrm{S}_{s}=2\left(c_{10}+c_{01}\right) \mathrm{F} .
$$

Combining the material response (deviatoric part) with the reactive stress (spherical part) coming from the incompressibility condition, the Cauchy stress tensor on the actual shape $\mathfrak{D}_{s}$ is given by

$$
\mathbf{T}_{s}=-p_{s} \mathbf{I}+2\left(c_{10}+c_{01}\right) \mathrm{FF}^{\top} .
$$

Herein we omit the reactive stress in the transversal direction: this part of the stress can be computed in the postprocessing and does not enter the equations we are writing down for the two-dimensional problem. The first term in (5) embodies inertial forces and is expressed as

$$
\int_{\mathcal{D}_{s}}-\rho_{s} \ddot{\mathrm{u}}_{s} \cdot \mathrm{w}_{s} d V
$$

where $\rho_{s}$ is the mass density of the solid material and $\mathrm{u}_{s}$ is the displacement vector field. The incompressibility condition is enforced in the weak form

$$
\int_{\mathcal{D}_{s}}(\operatorname{det} \mathrm{F}-1) \tilde{p}_{s} d V=0,
$$

in which $\tilde{p}_{s}$ is an appropriate test scalar field on $\mathcal{D}_{s}$.

\subsection{The fluid}

The balance principle for the fluid part states that the working

$$
\begin{aligned}
& \int_{\mathfrak{D}_{f}} \mathbf{b}_{f} \cdot \mathbf{w}_{f} d V+\int_{\partial_{\star} \mathfrak{D}_{f}} \mathbf{t}_{f} \cdot \mathbf{w}_{f} d A+ \\
& +\int_{\mathfrak{I}^{\prime}} \mathbf{t}_{f} \cdot \mathbf{w}_{f} d A-\int_{\mathfrak{D}_{f}} \mathbf{T}_{f} \cdot \nabla \mathbf{w}_{f} d V
\end{aligned}
$$


be zero for any test velocity field $\mathbf{w}_{f}$ on the actual shape $\mathfrak{D}_{f}$. The response function for a Newtonian fluid, together with the incompressibility condition, provides the following expression for the Cauchy stress

$$
\mathbf{T}_{f}=-p_{f} \mathbf{I}+2 \mu \operatorname{sym} \nabla \mathbf{v}_{f},
$$

where $\mathbf{v}_{f}$ is the fluid velocity field on $\mathfrak{D}_{f}, \mu$ is the viscosity and $p_{f}$ is the fluid pressure. Though the motion is confined inside a rigid container (the sclera), the interface between solid and fluid phases (the cortex) is deformable. This is why we used the socalled moving-grid formulation, also referred to as ALE method. This method consists in rewriting (15) on $\mathcal{D}_{f}$, as in (2), by using a virtual grid deformation $\gamma$ mapping $\mathcal{D}_{f}$ to $\mathfrak{D}_{f}$. The gradient of the fluid velocity field $\mathbf{v}_{f}$ on $\mathfrak{D}_{f}$ and the gradient of the fluid velocity field $\mathrm{v}_{f}$ on $\mathcal{D}_{f}$ turn out to be related by

$$
\nabla \mathbf{v}_{f}=\nabla \mathbf{v}_{f} \Gamma^{-1},
$$

where $\Gamma:=\nabla \gamma$ is the grid deformation gradient. The same relation applies to test velocity field gradients as well. Briefly, this domain transformation results in rewriting the last of the integrals in (15) as follows

$$
\int_{\mathcal{D}_{f}} \mathrm{~T}_{f} \Gamma^{-\mathrm{T}} \cdot \nabla \mathrm{w}_{f} \operatorname{det} \Gamma d V .
$$

Moreover, it is easy to show that the fluid particle acceleration on $\mathcal{D}_{f}$ is given by

$$
\mathrm{a}=\dot{\mathrm{v}}_{f}+\nabla \mathrm{v}_{f} \Gamma^{-1}\left(\mathrm{v}_{f}-\dot{\gamma}\right) .
$$

In details, the first term in (15) becomes

$\int_{\mathcal{D}_{f}}-\rho_{f}\left(\dot{\mathrm{v}}_{f}+\nabla \mathrm{v}_{f} \Gamma^{-1}\left(\mathrm{v}_{f}-\dot{\gamma}\right)\right) \operatorname{det} \Gamma \cdot \mathrm{w}_{f} d V$,

$\rho_{f}$ representing the fluid mass density, while the last term, through substitution of (16), results in

$$
\begin{gathered}
\int_{\mathcal{D}_{f}}\left[\left(-p_{f} \mathbf{I}+2 \mu \operatorname{sym}\left(\nabla \mathrm{v}_{f} \Gamma^{-1}\right)\right) \Gamma^{-\mathrm{T}} \operatorname{det} \Gamma\right] \\
\cdot \nabla \mathrm{w}_{f} d V .
\end{gathered}
$$

Finally we assign the incompressibility condition for the fluid in the weak form

$$
\int_{\mathfrak{D}_{f}}\left(\operatorname{div} \mathbf{v}_{f}\right) \tilde{p}_{f} d V=0,
$$

where $\tilde{p}_{f}$ is an appropriate test scalar field on $\mathfrak{D}_{f}$.

\subsection{Moving grid}

The model implemented in Comsol Multiphysics is made of three different application modes. Two of them are PDE Application Modes and contain the equations in weak form of both solid and fluid parts respectively. The equations are exactly those coming from the balance principles stated in the previous subsections. The last one is a Moving Mesh Application Mode, which allows us to avoid explicitly making use of expressions like (18), (20) and (21).

We leave Comsol perform such conversions by using the embedded algorithm for extending $\gamma$ from the boundary $\partial_{\star} \mathcal{D}_{f} \cup \mathcal{I}$ to the interior of $\mathcal{D}_{f}$, assuming that what it does is exactly what we mean. Nevertheless, we have to describe the boundary conditions on $\partial_{\star} \mathfrak{D}_{s}, \partial_{\star} \mathfrak{D}_{f}$ and across the interface $\mathfrak{I}$, defining at the same time the grid deformation $\gamma$. We assume that $\gamma$ matches the deformation of the solid part on $\partial_{\star} \mathfrak{D}_{s}$, on $\mathfrak{I}$ and on the interior of $\mathfrak{D}_{s}$. On the fluid side, we just assume that $\gamma$ matches the motion of $\partial \mathfrak{D}_{f}$. On the outermost boundary $\partial_{\star} \mathcal{D}$ of solid and fluid parts we assign a saccadic motion as a time varying rotation leaving the center of the eye $x_{0}$ fixed. We express the condition directly on the grid deformation $\gamma$ by giving the grid displacement field $\gamma(x)-x$ the following expression

$$
\mathrm{u}_{\gamma}=(\mathrm{R}-\mathrm{I})\left(\mathrm{x}-\mathrm{x}_{0}\right) .
$$

Further, we assume a no-slip condition on $\partial_{\star} \mathcal{D}_{f}$ in the weak form

$$
\int_{\partial_{\star} \mathcal{D}_{f}}\left(\mathrm{v}_{f}-\dot{\mathrm{u}}_{\gamma}\right) \cdot \tilde{\mathrm{t}}_{f} d A=0
$$

while on $\partial_{\star} \mathcal{D}_{f}$

$$
\mathrm{u}_{s}=\mathrm{u}_{\gamma} .
$$

Therefore the tractions $\mathrm{t}_{s}$ and $\mathrm{t}_{f}$ on the boundary $\partial_{\star} \mathcal{D}_{s} \cup \partial_{\star} \mathcal{D}_{f}$ are reactive.

\subsection{Interface}

The interface condition consists in the continuity of the velocity field across $\mathfrak{I}$

$$
\int_{\mathfrak{I}}\left(\mathbf{v}_{f}-\dot{\mathbf{u}}_{s}\right) \cdot \tilde{\mathbf{t}}^{*} d A=0
$$

where $\dot{\mathbf{u}}_{s}$ is the velocity field on $\mathfrak{D}_{s}$. By using the balance principle written separately 
for solid and fluid parts, together with the balance principle written for the whole body, we get to the conclusion that

$$
\begin{aligned}
& \int_{\mathfrak{I}} \mathbf{t}_{s} \cdot \mathbf{w}_{s} d A=-\int_{\mathfrak{I}} \mathbf{t}^{*} \cdot \mathbf{w}_{s} d A, \\
& \int_{\mathfrak{I}} \mathbf{t}_{f} \cdot \mathbf{w}_{f} d A=\int_{\mathfrak{I}} \mathbf{t}^{*} \cdot \mathbf{w}_{f} d A .
\end{aligned}
$$

Substitution of the previous expressions into (4) and (15) consists in replacing $\mathbf{t}_{f}$ with $\mathbf{t}^{*}$ and $\mathbf{t}_{s}$ with $-\mathbf{t}^{*}$. It is useful to summarize the expression we obtain for the integral over $\mathfrak{I}$ summing up terms in (4), (15) and $(26)$

$$
\int_{\mathfrak{I}} \mathbf{t}^{*} \cdot\left(\mathbf{w}_{f}-\mathbf{w}_{s}\right)+\left(\mathbf{v}_{f}-\dot{\mathbf{u}}_{s}\right) \cdot \tilde{\mathbf{t}}^{*} d A=0 .
$$

\section{Numerical Simulations}

The saccadic motion, which is a single rapid rotation of the eye, is usually approximated by using a five-degree polynomial whose graph is shown in figure 3 . We show the results of a simulation for a saccade of amplitude $A=10^{\circ}$ and duration $T=0.045 \mathrm{~s}$. The circular domain $\mathcal{D}$ has radius $R=0.012 \mathrm{~m}$. Material constants $c_{01}$ and $c_{10}$ for the solid response function $(7)$ are set to $50 \mathrm{~Pa}$, while the fluid viscosity $\mu$ is set to $10^{-3} \mathrm{~Pa}$ s. Solid and fluid mass densities are given the same value $\rho_{s}=\rho_{f}=1000 \mathrm{~kg} / \mathrm{m}^{3}$. The paragon shape $\mathcal{D}$ created in Comsol is rotated by $\pi / 4$ with respect to figure 2, The time integration procedure in Comsol required a fine tuning of the absolute tolerance in order to cover the all time interval $T$.

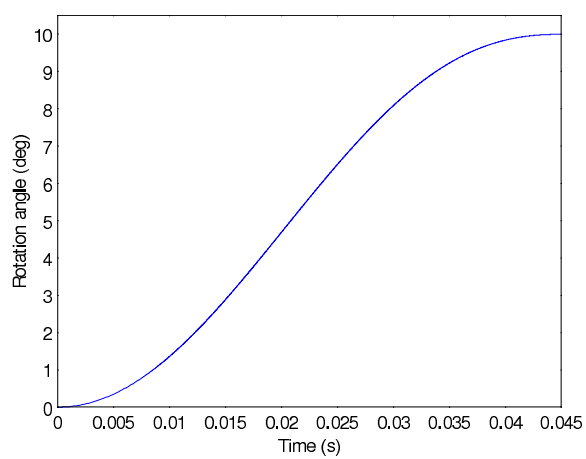

Figure 3: Rotation angle for a saccade of amplitude $A=10^{\circ}$.

In figures 4 and 5 we display the solid and fluid pressure fields together with the deformed shape of the interface at time $t=T$.

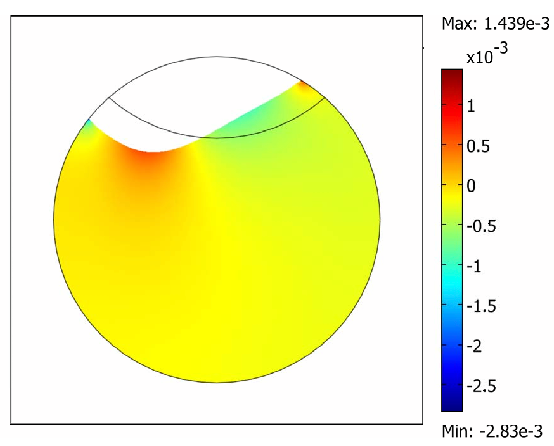

Figure 4: Pressure field $p_{s}$ on $\mathfrak{D}_{s}$ at time $t=0.045 \mathrm{~s}$.

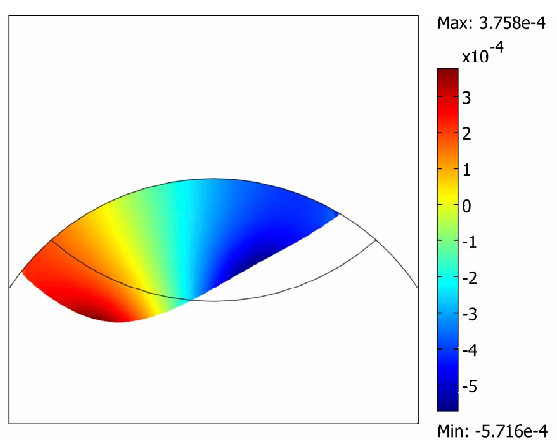

Figure 5: Pressure field $p_{f}$ on $\mathfrak{D}_{f}$ at time $t=0.045 \mathrm{~s}$.

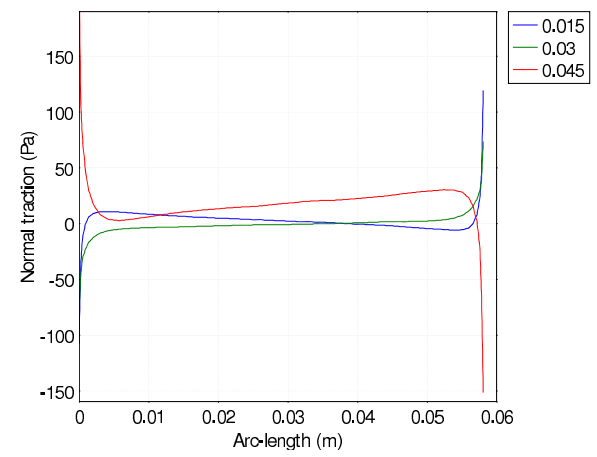

Figure 6: Normal traction on $\partial_{\star} \mathfrak{D}_{s}$ at different times as in the legend.

Normal and tangential components of the traction $\mathbf{t}_{s}$ are shown in figures 6 and 7 : at the contact points between the interface $\mathfrak{I}$ and the solid boundary $\partial_{\star} \mathfrak{D}_{s}$ (corresponding to the boundary of the adhesion area between the vitreous and the retina), high values of the traction are attained during the saccadic movement. In particular, during the rotation both the components of the traction increase from an initial zero value attaining a high value, and they decrease afterwards until they reach an opposite still 
high value. The rupture in the retina can be induced by positive high values of the normal traction and by high values of the tangential traction, whether positive or negative.

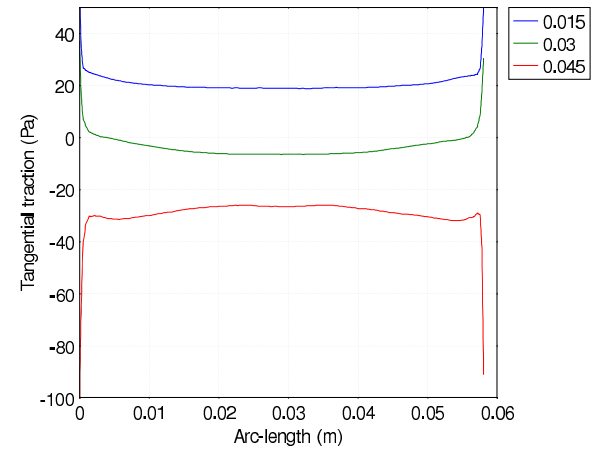

Figure 7: Tangential traction on $\partial_{\star} \mathfrak{D}_{s}$ at different times as in the legend.

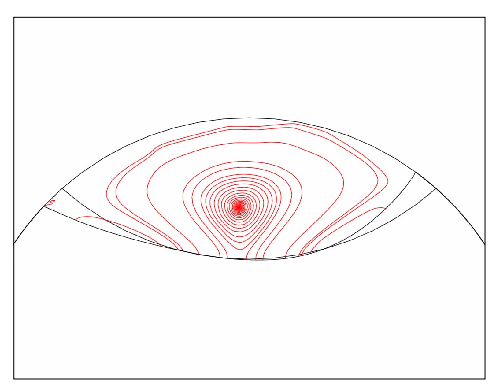

Figure 8: Streamlines on $\mathfrak{D}_{f}$ at time $t=0.022 \mathrm{~s}$.

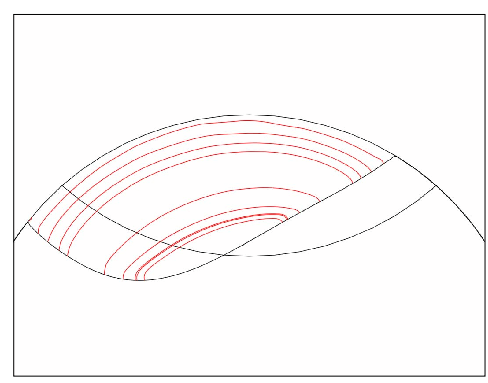

Figure 9: Streamlines on $\mathfrak{D}_{f}$ at time $t=0.045 \mathrm{~s}$.
In the end we can note the complexity of the liquefied vitreous motion, showing the relative velocity field by using streamline plots (figures 8, 9).

\section{References}

[1] P.L. Kaufman and A. Alm, Adler's physiology of the eye, Mosby, 2003.

[2] B. Lee, M. Litt, and G. Buchsbaum, Rheology of the vitreous body. Part I: viscoelasticity of human vitreous, Biorheology 29 (1992), 521-533.

[3] R.W. Ogden, Non-linear elastic deformations, Dover Publications, Inc., 1997.

[4] J. Sebag, The vitreous: Structure, function and pathobiology, Springer and Verlag, 1989.

[5] J. Sebag and E.A. Balasz, Morphology and ultrastructure of human vitreous structure, Investigative Ophthalmology and Visual Science 30 (1989), no. 8, 1867-1871.

[6] P. Le Tallec, Handbook of numerical analysis, vol. 3, Elsevier Science, 1994.

[7] C. Truesdell, A first course in rational continuum mechanics, vol.1, Academic Press, 1977.

[8] J. Wang, D. McLeod, D.B. Henson, and P.N. Bishop, Age-dependent changes in the basal retinovitreous adhesion, Investigative Ophthalmology and Visual Science 44 (2004), no. 5, 1793-1800. 